\title{
Dutch National Institute for Sports Health Care
}

On 30 March 1981 the Netherlands Association of Sports Medicine (NASM), the Federation of Bureaux for Sports-medical Examination (FBSE), the Netherlands Sports Federation (NSF) and the Royal Netherlands Football Association (RNFA) decided to found the National Institute for Sports Health Care (NISHC), and to appoint a provisional Board.

This decision was the logical outcome of plans fostered by the NASM as early as 1968 to establish what was then called a national sports-medical centre. In 1972 these plans were further elaborated in the report 'Advice on Sports-medical Guidance', published by the Public Health Council. In 1975 the NASM presented its policy plan 'The Future of Sports Medicine', of which an Institute for Sports Medicine was an essential feature. At the general annual meeting of the NSF in 1975, the associations affiliated with the NSF unanimously accepted this policy plan as a basis for a national system of sports health care.

The FBSE, RNFA, NSF and NASM collaborated intensively to establish a National Institute for Sports Health Care.

In 1980 the FBSE and the NASM opted for further intensification of their joint efforts, partly in order to incorporate the Sports-medical Guidance Centres (SGCs/SMAs) and Bureaux for Sports-medical Examination fully in the NISHC. It was agreed that the FBSE, as national coordinating organization, would ultimately discontinue its activities, while the NASM would transfer many of its national tasks to the new Institute. This decision was based in part on a study performed by Berenschot at the request of the FBSE and NASM, and the resulting report 'Structures in Sports Health Care' which was accepted at the annual meetings of the FBSE and the NASM on 31 January 1981. Both general meetings also decided to found 'an organization for sports health care', and to incorporate the activities of the Sports-medical Guidance Centres and of the Bureaux for Sports-medical Examination in such an organization, stipulating that the regional SGC and the BSE (Bureaux) active in the corresponding regions would form regional units, to be affiliated to the national organization.

The organization of some 30 SGC and over 200 BSE in regional units required careful management, particularly in view of the slightly different executive structures and histories in the different regions. It was therefore very fortunate that - as with the report 'Structures in Sports Health Care' - the Ministry of Culture, Recreation and Social Work (CRSW) was again prepared to finance a study by a team of management consultants. This was intended to lead to a model regional structure in sports health care, with possible

(C) 1989 Butterworth \& Co (Publishers) Ltd 0306-3674/89/040222-04\$03.00 variants, also in relation to a future organizational structure for sports health care on a national level.

Shortly after this study had been ordered, the smoothly conducted consultations between FBSE, NASM, NSF and RNFA led to the decision to found the NISHC. The fact that this decision was made earlier than could have been expected at the turn of the year $(1980 / 1981)$ is explained by several factors, including some organizational and financial considerations. Primarily, however, this decision was based on the desire of the organizations involved to make sports health care of good quality available to every Dutch sportsman. The rapid evolution of sports health care however welcome as such - threatened to confuse the average sportsman's notions regarding the facilities available. Particularly for this reason there was an urgent need for an institute which, with the consent of and in colaboration with the partners involved, could direct and guide national, regional and local activities in sports health care.

In view of the importance of coordination of the regional and the national structure, representation of regional units on the provisional Board was immediately ensured. In actual practice this meant that the BSE were still represented by the executive committee of the FBSE, and that the SGC appointed their own immediate representative on the Board. This also demonstrated the earnest intention of the NASM to effect complete transfer of its formal responsibility for the activities of the SGC to the NISHC as soon as possible.

The draft articles of association defined as tasks for the National Institute of Sports Health Care:

- education, information and documentation

- consultation on a national level

- coordination of regional and national activities

- advice and guidance, in the widest sense, of the sports-medical regional units

- advice and coordination of, and possibly participation in, medical guidance within the sports organizations, and the development of guidelines

- development, organization and evaluation of training courses, and adjustment of training programmes for medical and/or para-medical functions

- definition and control of quality standards for the organization and for medical and/or paramedical treatment

- initiation, cataloguing, coordination, registration and execution (or guiding the execution) of research projects

- all other legitimate activities which can lead to achievement of the goal.

Representation on the Board of the NISHC includes members from NASM, NSF, FBSE, SGC and RNFA. The NISHC started off with a staff of six; in 1989 this has become sixteen. The main tasks are:

- furtherance of scientific research

- improvement of sports-medical guidance

- information 

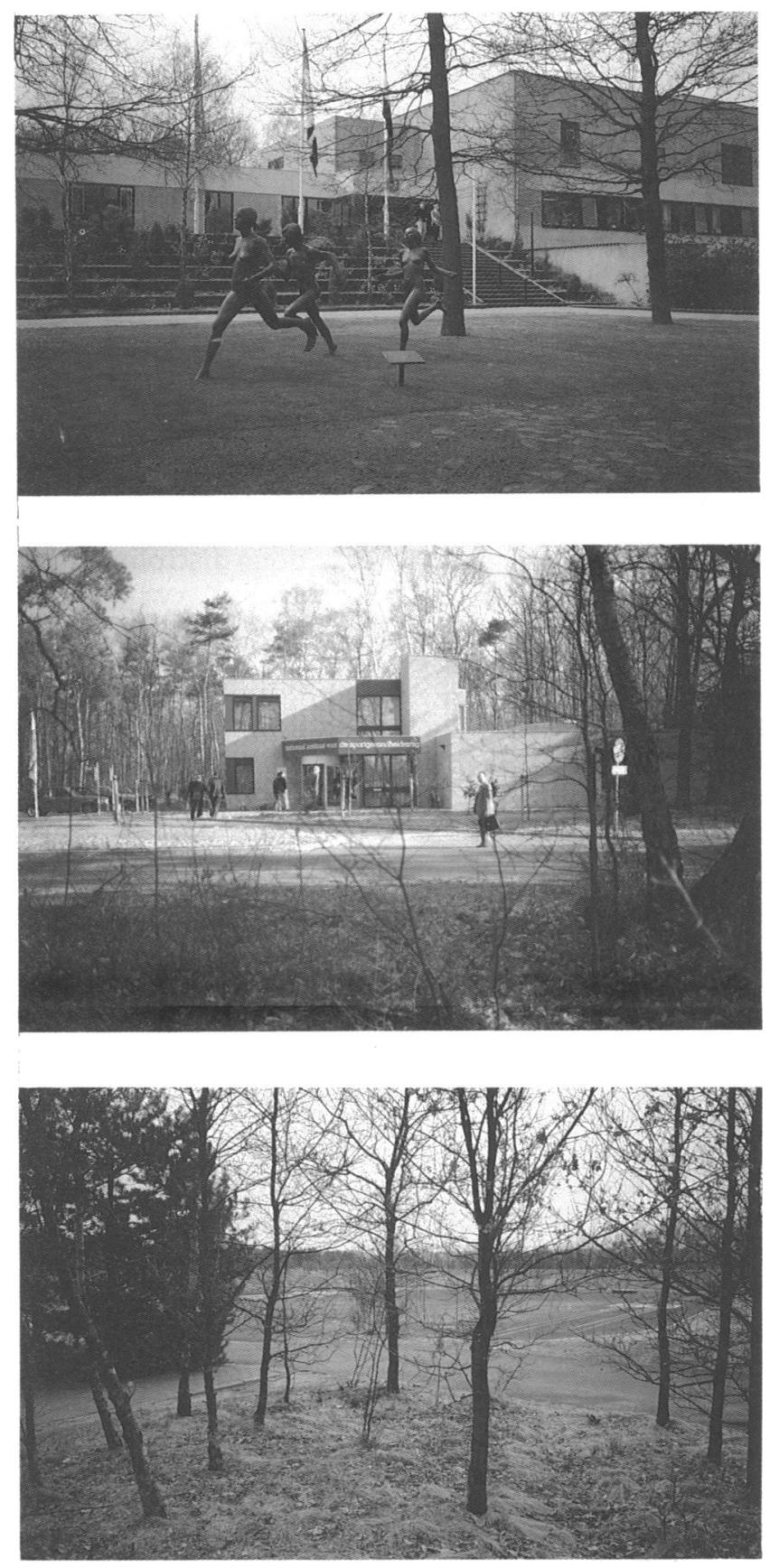

\section{Scientific research}

The NISCH has initiated a Scientific Board in which all the universities with a medical faculty participate. This board reviews proposals and outcomes of scientific research and produces a survey of research in progress. Furthermore, the NISCH participates in the Janus Jongbloed Research Centre, which - sponsored by a bank - plays an important role in conducting research and in introducing sports medicine in several fields of study.

\section{Sports medical guidance}

Holland has about 40 centres of sports medical advice, so called SMAs. These centres are set up apart from the regular health care service and run by general prac- titioners and physiotherapists who take a special interest in sportsmedical advice. The NISHC operates as a master organization for these SMAs, subsidizes their activities and assists with guidelines, materials and educational support. There are also two centres for sports medical guidance and revalidation where fulltime specialists take care of top-level sportsmen.

\section{Information}

Information to sportsmen is given by the SMAs on a personal basis and by the NISHC through various publications.

A major campaign was launched in 1989 to prevent sports injuries. With the slogan: 'Injuries, keep ahead of them' in widespread national publicity, with various campaign materials and with special regional and local activities, the organization is trying to reduce the approximately half a million sports injuries annually that need medical attention.

\section{Education}

Most medical students in Holland still give only marginal attention to sports medicine as a special discipline. There is some hope this may change in the years to come as sports medicine in 1986 has been officially registered as a specialization. At present, the NISHC presents annually a broad programme of sports medical courses for GPs and medical specialists.

The history of education and training in sports medicine in the Netherlands is still short. It largely coincides with the history of the Netherlands Association of Sports Medicine, whose founders in 1960 organized the first applied course on sports medicine to offer postgraduate training to physicians interested in sports medicine.

Within the Association of Sports Medicine the conviction grew that daily sports medical problems should be solved by the existing health care agencies, i.e. by general practitioners and hospital specialists. Utilization of existing medical facilities is a practical necessity in view of the mass of active sportsmen (4.2 million in a population of about 13 million) and the resulting mass of sports medical problems. For this purpose, the existing medical facilities should be adequately prepared; that is to say, the knowledge of sports medicine required to cope with the everyday problems should be available.

In addition, both the world of medicine and the world of sport will need several physicians engaged full-time in sports medicine and with specialized knowledge of this discipline. Between the physician incidentally confronted by sports medical problems and the full-time specialist in sports medicine, there is a need for a group of physicians who practice sports medicine, not incidentally, but regularly as a part-time activity. Such activity may be the guidance of a sports medical consultation centre or the guidance of groups of sportsmen during training and events, in part-time functions like also that of club or federation doctor. This group of part-timers, who shoulder the main burden of sports medical work, should be equipped with specific knowledge for this purpose. 
NASM envisaged three different training levels: one for physicians incidentally confronted with sports medicine in daily practice; a second one for those who regularly occupy themselves with sports medicine in a part-time function; and a third for a group of full-time specialists with positions in health care or in sports.

\section{First level training}

For the first-level training to be achieved - giving every graduate physician some knowledge of sports medicine - relevant instruction should be included in the university curriculum. This does not mean a separate chair for sports medicine, but rather facilities within every medical discipline for instruction in the sports medical aspects of that particular discipline. The physiologist should focus on the physiology of exertion, for example, and the orthopaedist should discuss the traumatology of sports. Like all changes, however, this process takes a considerable amount of time, especially in an academic world not renowned for its craving for innovation.

\section{Second level training}

The second level of training concerns the part-timers: the group of physicians active in sports medicine on a part-time basis. For example, physicians who:

- direct sports medical boards

- provide consultation on sports at schools

- work at a sports medical consultation centre (SMA)

- are engaged by federations and major clubs to give regular medical care and guidance to teams and groups of sportsmen.

Training provided for this group has been organized in the form of an applied course: graduate or specialized physicians can attend an 80 hour course over two consecutive weeks to gain more detailed knowledge about sports orthopaedics and traumatology, sports physiology, cardiology and general sports medical problems such as nutrition, doping, medication, influence of climate factors, practice of medical guidance etc.

In the past 16 years these courses, provided by the Association of Sports Medicine, have been much appreciated by physicians and specialists in day-to-day practice. A maximum of 30 persons attend each course, i.e. a total of 60 per year. Courses have been fully booked at least a year in advance without any canvassing activities.

The substance and form of the course are constantly kept up-to-date on the basis of regular evaluation by instructors and course members, and with the aid of professional university institutes.

\section{Non-medical courses}

Apart from the applied courses for physicians, there are postgraduate courses for paramedical personnel and particularly for physiotherapists. These courses, too, are popular and have waiting lists. The practical organization of these courses was initially impeded by the need for capable instructors experienced in the practice of sports medicine.

\section{Specialist sports medicine training}

The training of full-time specialists in sports medicine has likewise been given form and substance. Given the current level of organization of Sports Medicine in the Netherlands, it was estimated that 25-30 specialists of this kind would be needed early on. The objective of specialization in sports medicine is to provide specialists who can cope with all sports medical problems in fitness examination, prevention and guidance and who, in addition, are able to evolve problem statements and elaborate research projects in cooperation with specialists in various disciplines and scientific university institutes. This four-year training programme - provided in the form of residencies comprises the following elements:

- 1 year of physiology

- 1 year of cardiology

- 1 year of orthopaedics

- 1 year of general sports medicine.

\section{Physiology}

The objective of the physiological year in the training of sports physicians is to provide some basic knowledge of physiology, experience in research, knowledge of and experience with measuring techniques and methods of investigation, particularly those used in the physiology of exertion. Finally, there is experience in the use of certain statistical methods. This part of the course takes a year and consists of a period of practical training at a physiological laboratory or equivalent institute. If possible, a test paper is written on a subject determined in consultation with the instructor.

\section{Cardiology}

The objective of the cardiological/pulmonological year in the training programme is to familiarize the physician with methods of investigation and clinical and therapeutic work in the field of cardiology and pulmonology, enabling him to identify and cope with the problems he is likely to encounter in later practice, and to solve these problems (or have them solved by qualified experts). At least five months of this year are spent in a function unit of a cardiological department, and at least another five months in a clinical ward. The remaining months are devoted to possible gaps in training or to a more detailed study of certain aspects, e.g. child cardiology or lung function laboratory. In the clinical as well as in the functional training periods, specific sports-medical problems receive ample attention. Again, a test paper is afterwards written on a subject agreed with the instructors.

\section{Orthopaedics}

The objective of this part of the programme is to acquire knowledge of the diagnosis and treatment of the most common orthopaedic and traumatological conditions, with ample opportunity to get acquainted with 
specific sports injuries and their treatment. This period is therefore spent in a department which ensures a constant supply of suitable cases.

The resident-trainee works both at the out-patient clinic and in the wards, and should acquire skills in general orthopaedic examination, diagnostic radiology, indications for physiotherapeutic and pharmaceutical measures, and problems of postoperative treatment. In addition he works at a first-aid station in order to gain experience in the treatment of simple wounds, the diagnosis of fractures, and diagnostic radiology. Much additional attention is focused on contacts with physiotherapy departments to ensure that the trainee acquires sufficient knowledge of methods of physiotherapy and their indications to enable him to cooperate effectively with physiotherapists in the future.

\section{General sports medicine}

In the year of training in general sports medicine the future sports physician learns to apply his previously acquired knowledge of cardiology, physiology and orthopaedics in the practice of sports medicine, e.g. by direct guidance of individuals and teams in training and competition. In addition, a period of practical training with a general practitioner is completed during this year.

The practical training is received in the sports-medical departments of the Netherlands Sports Federation and the Royal Netherlands Football Association, where several full-time sports physicians supervise the trainee.

This training programme, which has evolved to a more or less definitive form, in highly detailed, registered training requirements per discipline, is still subject to evolution. Both the instructors and the trainees attach great importance to regular evaluation of the programme so that, if necessary, modifications can be made.

In organizational terms, the training of specialized sports physicians is made possible by the Foundation for the Training of Sports Physicians (SOS), which partly acts as financing institute but also contributes to the organizational work. Both sports organizations and medical organizations are therefore represented in this Foundation. The participant organizations are:

- The Royal Netherlands Medical Association

- The Netherlands Association of Sports Medicine

- The Netherlands Heart Foundation

- The Netherlands Sports Federation

- The Royal Netherlands Football Association.

The Foundation for the Training of Sports Physicians is assisted by a council of specialists in physiology, cardiology, orthopaedics and general sports medicine; and this council determines and controls the actual form and substance of the training programme.

Completed training is officially registered by a Registration Board on which the Royal Netherlands Medical Association is represented. This registration finally leads to a qualification as a sports physician a specialism for which the Royal Netherlands Medical Association, in consultation with the Association of Sports Medicine, created a special place in 1986 within the system of medical qualifications. The Central Committee of the Foundation for the Training of Sports Physicians acts as Board of Appeal during the training and qualification procedure.

\section{Authority of NISHC}

Over the years, the NISHC has become an important body for consultation and policy in the field of sports medicine. Its authority is widely respected and some related organizations have moved to the NISHCbureau: the Netherlands Association of Physiotherapy in Sports Health Care and the recently founded Netherlands Centre for Doping Problems. The NISHC is also the stimulating factor in the Council of Europe meetings on sports medicine.

Finances are still the bottleneck for exercising various activities. The NISHC is partly financed by the government and partly has to take care of its own sources of income, mainly by sports medical courses and rendering of specific services.

For detailed information please contact: Dutch National Institute for Sports Health Care, P.O. Box 90 6860 AB Oosterbeek, The Netherlands. Tel. +31.8308 .1984 Telefax +31.8308 .1952 . 